\title{
THE HOMOLOGICAL DIMENSIONS OF SYMMETRIC ALGEBRAS
}

BY

\author{
JAMES E. CARRIG(')
}

\begin{abstract}
Let $D$ be a Dedekind domain and $M$ a rank-one torsion-free $D$-module. An analysis of $A=S_{D}(M)$, the symmetric algebra of $M$, yields the following information:

Throrem. (1) Tor-dim $A<2$ and $=1$ iff $M=K$, the quotient field of $D$;

(2) $A$ is coherent;

(3) Global $\operatorname{dim} A=2$.

For higher rank modules coherence is not assured and only rough estimates of the dimensions are found.

On the other hand, if $S_{D}(M)$ is a domain of global dimension two, then $M$ has rank one but the dimension of $D$ may be two. If $D$ is local of dimension two then $M=K$.
\end{abstract}

0. Introduction. The aim of this paper is to study the homological dimensions of symmetric algebras. For reasons that will become obvious these algebras present far more difficulties than group algebras of abelian groups, for example, especially as the dimensions increase. For the most part, we shall concern ourselves with the study of dimension two and focus on the pivotal role played by the symmetric algebra of a rank one flat module over a Dedekind domain. This algebra is shown to be coherent of the required dimension. Unfortunately, the vital property of coherence disappears for higher rank modules and we must rely on cardinality conditions to give estimates of the dimensions.

We next take up the reverse question by attempting to describe the symmetric algebras of dimension two. Although a complete description is not given, enough information is obtained to provide a reasonably clear picture, especially in the case of domains where they are fully characterized.

1. Dimensions of symmetric algebras. All rings are assumed commutative and we shall follow the staridard terminology as in [1], [2] and [7], except that a local ring will not necessarily be Noetherian. In addition the weak global

Received by the editors March 30, 1976.

AMS (MOS) subject classifications (1970). Primary 13D05.

Key words and phrases. Global dimension, Tor-dimension, coherent, symmetric algebra, Dedekind domain, rank one flat module.

(l) This work constitutes a portion of the author's $\mathrm{Ph}$.D. dissertation written under the direction of Professor Wolmer V. Vasconcelos.

- American Mathematical Society 1978 
dimension of a ring $A$ is denoted here by Tor-dim $A$.

We begin by determining the Tor-dimension of the symmetric algebra of a rank one flat module over a Noetherian ring of finite global dimension.

(1.1) Leman. Let $A$ be a G.C.D. domain and $M$ a rank-one flat module. Then $M$ is the directed union of cyclic submodules.

Proof. We may assume $M \subseteq K=q f(A)$. It is enough to show that the cyclic submodules of $M$ form a directed system.

Let $\alpha, \beta \in M, \alpha=a^{\prime} / b^{\prime}, \beta=c^{\prime} / d^{\prime}$. Since $A$ is a G.C.D. domain we can assume

$$
\operatorname{gcd}\left(a^{\prime}, b^{\prime}\right)=1=\operatorname{gcd}\left(c^{\prime}, d^{\prime}\right) .
$$

Let $e=\operatorname{gcd}\left(a^{\prime}, c^{\prime}\right), f=\operatorname{gcd}\left(b^{\prime}, d^{\prime}\right)$ and rewrite $\alpha=e a / f b, \beta=e c / f d$ with $\operatorname{gcd}(b, d)=1=\operatorname{gcd}(a, c)$.

Since $b c \alpha=a d \beta$ we have the following relation:

$$
b c \alpha-a d \beta=0 .
$$

By the flatness of $M$ there exists $\alpha_{j} \in M$ and $b_{j}, c_{j} \in A$ such that

$$
\alpha=\sum b_{j} \alpha_{j}, \quad \beta=\sum c_{j} \alpha_{j}
$$

and $b c b_{j}=a d c_{j}$ for all $j$. Since $\operatorname{gcd}(a, b)=\operatorname{gcd}(a, c)=1$, this forces $a \mid b_{j}$ hence $b_{j}=a b_{j}^{\prime}$ and so $b c b_{j}^{\prime}=d c_{j}$. Similarly we get $b_{j}^{\prime}=d b_{j}^{\prime \prime}$ and hence $b c b_{j}^{\prime \prime}=c_{j}$. Combining we have $\alpha=a d \Sigma b_{j}^{\prime \prime} \alpha_{j}$ and $\beta=b c \Sigma b_{j}^{\prime \prime} \alpha_{j}$. Thus the cyclic submodules are directed and the result follows.

An easy application gives us information on the Tor-dimension.

(1.2) Proposmon. Let $A$ be locally a G.C.D. domain and $M$ a rank-one flat module. Then

$$
\text { Tor-dim } S(M)<\text { Tor-dim } A+1 \text {. }
$$

Proof. Since the Tor-dimension is determined locally, we may assume $A$ is local. By (1.1) $M$ is then the union $M=\cup M_{\alpha}$ of cyclic submodules. We write

$$
S(M)=\text { ind } \lim S\left(M_{\alpha}\right)=\operatorname{ind} \lim A\left[x_{\alpha}\right]
$$

since each $M_{\alpha}$ is in fact free on one generator. The flat version of Hilbert's syzygies theorem gives

$$
\text { Tor-dim } A\left[x_{\alpha}\right]=1+\text { Tor-dim } A,
$$

and the result is then clear.

We may make a more precise statement about Prüfer domains:

(1.3) Propostrion. If $A$ is a Prüfer domain and $M$ a rank-one flat A-module, then

(i) Tor-dim $S(M)<2$, 
(ii) Tor-dim $S(M)=1$ if and only if $M=K$, the quotient field of $A$.

Proof. (i) If $A$ is Prüfer it has Tor-dimension one and is locally a valuation ring which is a G.C.D. domain. Apply 1.2.

(ii) We view $S(M)$ as the subring of $K[t]$ given by

$$
S(M)=A+M t+M^{2} t^{2}+\ldots
$$

If Tor-dim $A=1$, then for every $0 \neq a \in A$ the ideal $(a,(M t))$ is flat and properly contains the prime ideal $(M t)$. The next step is contained in a lemma.

(1.4) LEMaMA. If a flat ideal $I$ in a ring $A$ properly contains the prime ideal $P$ then $P=P I$.

Proof. Since $I$ is a flat $A$-module we tensor by $A / P$ to get $I / P I$, a flat $A / P$-module. As $A / P$ is a domain $I / P I$ is torsion-free. Consider the sequence

$$
0 \rightarrow I \rightarrow A \rightarrow A / I \rightarrow 0
$$

and tensor by $A / P$ to get

$$
0 \rightarrow \operatorname{Tor}_{1}(A / I, A / P) \rightarrow I / P I \rightarrow A / P \rightarrow A / I \rightarrow 0 .
$$

Now $\operatorname{Tor}_{1}(A / I, A / P) \approx I \cap P / P I=P / P I$. If $I / P I$ is torsion-free, so is $\operatorname{Tor}_{1}(A / I, A / P)$ as it is a submodule. But $I / P$ annihilates $\operatorname{Tor}_{1}(A / I, A / P)$, thus forcing it to be trivial. Hence $P / P I=0$ and $P=P I$.

Returning now to the proof of (1.3) we see from the lemma that $(a,(M t))(M t)=(M t)$ and hence $a M=M$ for all $a \in A$. This shows that $M$ is divisible and thus $M=K$.

To see the reverse implication we write

$$
B=S(K)=A+K t+K t^{2}+\ldots
$$

First we note that if $I$ is an ideal of $B$ such that $I \cap A=J \neq 0$ then $I=J B$.

Now let $I=\left\{f_{1} \cdots f_{n}\right\}$ be a finitely generated ideal of $B$, and we show that it is projective.

If $I \cap A=J \neq 0$, then $J$ is a finitely generated ideal of $A$ and thus projective. This forces $I=J B$ to be $B$-projective. Thus we may assume $I \cap A=0$. As $B_{A-\{0\}}=K[t], I_{A-\{0\}}$ is principal, generated by an element of least degree which may be taken to be one of the $f_{j}$, say $f_{1}$. Then for all $k$, $f_{k}=g_{k} f_{1}$ where $g_{k} \in K[t]$. If $0 \neq f_{1}(0)=a \in A$ we may write

$$
f_{k}=f_{1}\left(a g_{k}\right) / a \text { for all } k
$$

with $h=f_{1} / a \in B$ and $h(0)=1$, forcing $a g_{k} \in B$.

If $f_{1}(0)=0$, then we may write $f_{k}=f_{1}\left(b g_{k}\right) / b$ where $b \in A$ such that $b g_{k} \in B$ for all $k$, and $h=f_{1} / b \in B$. 
In either case we note that

$$
I=h L \quad \text { where } L \cap A \neq 0 .
$$

As $L=(L \cap A) B, L$ is then a projective $B$-module and hence $I=h L$ is also projective.

For the rest of this section we make the blanket assumption that $D$ is a Dedekind domain, $M$ a rank-one torsion-free (hence flat) $D$-module, and set $A=S_{D}(M)$. We remark that for primes of $D, M_{P}$ is either cyclic or equal to $K$, the quotient field of $D$.

For nonfinitely generated $M$, the ring $A$ is not Noetherian, nor in general locally Noetherian. To check this however, we need only check at the primes of $D$ :

(1.5) LEMMA. $A=S_{D}(M)$ is locally Noetherian at primes of $A$ if and only if it is so at primes of $D$.

Proof. Clear.

The following lemma will prove useful in our discussion of coherence:

(1.6) LEMMA. If I is a nonzero ideal of $D$ then $A / I A$ is Noetherian.

Proof. We have $A / I A=S_{D / I}(M / I M)$. Since $D / I$ is Artinian we only need show that $M / I M$ is finitely generated.

Write $D / I=D_{1} \oplus \ldots \oplus D_{n}$ where the $D_{j}$ are Artin local rings and express

$$
S_{D / I}(M / I M)=\bigoplus S_{D_{j}}\left(D_{j}(M / I M)\right) \text {. }
$$

It is then sufficient to show that $M_{j}=D_{j}(M / I M)$ is finitely generated for all j.

Since $M$ is $D$-flat, $M / I M$ is $D / I$-flat and hence $M_{j}=D_{j}(M / I M)$ is $D_{j}$-flat. An appeal to [2] shows that $M_{j}$ are $D_{j}$-free. However, since $M$ is rank-one, $\wedge^{2} M=0$ which gives that $\wedge^{2} M / I M=0$ which in turn implies that $\wedge^{2} M_{j}=0$. But if the $M_{j}$ are free, rank considerations force $M_{j}$ to be rank-one. Putting the pieces together we get $M / I M$ finitely generated, whence the assertion.

(1.7) THEOREM. $A=S(M)$ is a coherent domain.

Proof. Let $I$ be a finitely generated ideal of $A$. Since $A$ is the directed union $A=\cup_{\alpha} S\left(M_{\alpha}\right)$ where the $M_{\alpha}$ are finitely generated, we may write $I=I_{0} A$ where $I_{0}$ is a finitely generated ideal of $A_{0}=S\left(M_{\beta}\right)$ for some $\beta$. $A_{0}$ is then Noetherian of dimension two. This last is true, since, by (1.5) we check at the localizations at primes of $D$, where $\left(M_{\beta}\right)_{p}$ is a free $D_{p}$-module of rank-one.

According to [12] we may express $I_{0}=K L_{0}$ in $A_{0}$ where $K$ is projective and 
$L_{0}$ is an ideal of grade two. As $K$ is finitely presented it is then enough to show that $L=L_{0} A$ is finitely presented.

First we note that $L_{0} \cap D=J \neq 0$ for, otherwise, in the localization at $D-\{0\}, L_{0}$ survives and has grade two in the ring $K[t]$, which is impossible.

Consider then the exact sequence

$$
0 \rightarrow J A \rightarrow L_{0} A \rightarrow L_{0} A / J A \rightarrow 0 .
$$

By the lemma $A / J A$ is Noetherian, hence $L_{0} A / J A$ is a finitely presented $A / J A$ ideal. $A / J A$ is in turn $A$-finitely presented and thus $L_{0} A / J A$ is $A$-finitely presented. As $J$ is an ideal of $D$ it is projective, so $J A$ is $A$-projective and hence $J A$ is finitely presented.

With both $L_{0} A / J A$ and $J A$ finitely presented, we have that $L_{0} A$ is finitely presented.

Remark. Though the Dedekind assumption may seem too strong at this point, in the next section we offer an example which shows that Prüfer is not enough.

Unfortunately, for higher rank modules coherence fails in general. However, with some cardinality conditions we can determine the dimensions as in the following example.

(1.8) EXAMPLE. Let $D=Z, M=Q \oplus Q$ and $A=S(Q \oplus Q)$. $A$ may be realized as

$$
A=Z+Q x+Q y+\ldots
$$

Since $(A x: A y)=(Q x)$, which is not finitely generated, we have that $A$ is not coherent. Nevertheless, we can determine the dimensions of $A$ as follows:

$$
\begin{aligned}
s(Q \oplus Q) & =s(Q)_{z} \otimes s(Q) \\
& =s(Q)_{z} \otimes \text { ind } \lim Z\left[x_{\alpha}\right]=\text { ind } \lim s(Q)\left[x_{\alpha}\right]
\end{aligned}
$$

which by (1.3) gives Tor-dim $A<2$. Localization at $Z-\{0\}$ gives Tor-dim $A$ $=2$. By Jensen's lemma [6] we get

$$
\text { gl } \operatorname{dim} A<3 \text {. }
$$

As domains of global dimension two are coherent [10], we have that

$$
\mathrm{gl} \operatorname{dim} S(Q \oplus Q)=3 \text {. }
$$

Similarly for higher dimensional analogs we have that

$$
\text { Tor-dim } S\left(Q^{n}\right)=n \text {, }
$$

but only gl $\operatorname{dim} S\left(Q^{n}\right)<n+1$.

Probably the higher bound holds.

Note that, at this point, we still have no bound on the global dimension without the use of cardinality conditions. Thus we turn our attention to that task.

Recall that if $D$ is a Dedekind domain and $M$ is a rank-one flat $D$-module, 
then $M_{p}=K$ or $M_{p}=(\alpha)$ for every prime $p$ of $D$. We are thus led to consider the following partition of the spectrum of $D$ :

$$
\begin{aligned}
& \mathscr{P}_{1}=\left\{P \in \operatorname{Spec} D \mid M_{P}=K\right\}=\{P \in \operatorname{Spec} D \mid P M=M\}, \\
& \mathscr{P}_{2}=\left\{P \in \operatorname{Spec} D \mid M_{P}=(\alpha)\right\}=\{P \in \operatorname{Spec} D \mid P M \neq M\} .
\end{aligned}
$$

Let $S_{1}=D-\cup_{P \in \Phi_{1}} P$ and $S_{2}=D-\cup_{P \in \Phi_{2}} P$. Thus $S_{1}$ and $S_{2}$ are multiplicative sets. Let

$$
D_{S_{1}}=D_{1} \text { and } D_{S_{2}}=D_{2}
$$

It is clear that $D_{1}$ and $D_{2}$ remain Dedekind domains. Hence the primes of $D_{1}$ correspond to primes in $\mathscr{P}_{1}$ and likewise for $D_{2}$. We then have $D_{1} M=K$ and $D_{2} M=M$. It is clear that the injection

$$
D \rightarrow D_{1} \times D_{2}
$$

is faithfully flat, hence the corresponding extension

$$
S_{D}(M) \rightarrow S_{D_{1}}(K) \times S_{D_{2}}(M)
$$

is also faithfully flat.

By the descent theorem [5], it is enough to find the global dimension of each of the components.

We first consider $A=S_{D_{1}}(K)=D_{1}+x K[x]$ and we have the Cartesian square

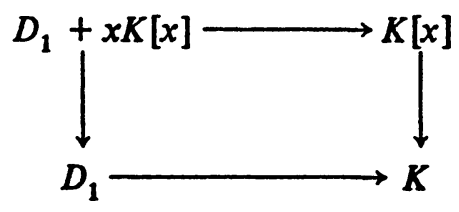

This situation conforms to [4] and we conclude

$$
\text { gl } \operatorname{dim} S_{D_{1}}(K)=2 \text {. }
$$

Hence it suffices to give the dimension of $A=S_{D_{2}}(M)$ which is locally Noetherian and we drop the subscript. Since Tor-dim $A=2$ and $A$ is coherent, finitely generated ideals have projective dimension at most one and the following proposition will take care of flat ideals.

(1.9) Proposition. If $I$ is a flat ideal of $A=S(M)$ then $\mathrm{pd} I<1$.

Proof. Let $I$ be a flat ideal and $J=\left\{a_{1} \ldots a_{n}\right\} \subseteq I$ a finitely generated subideal. $J$ admits a decomposition $J=M L$ where $M$ is projective and $L^{-1}=A$. We claim that $M \subseteq I$ and as a consequence $I$ is the directed union of finitely generated projective ideals.

It suffices to check this inclusion locally. As $I$ is flat and $A$ is locally a regular local ring of dimension two, $I_{p}=(f)$ a free ideal. On the other hand 


$$
J_{p}=M_{p} L_{p}=(g) L_{p}
$$

since $M_{p}$ is locally free. In fact $g$ is the gcd of the generators of $J_{p}$. Since $J_{p} \subseteq(f) f$ divides the generators and hence $(g) \subseteq(f)$. Thus $M \subseteq I$.

Now $I_{D-\{0\}}=(f)$ and $f$ may be taken in $I$. We now view $I$ as the directed union of the finitely generated projective subideals which contain $f$. For any such subideal $P, P^{-1} f$ is an invertible ideal of $A$. It is clear that $P^{-1} f \cap D=$ $N \neq 0$ and in fact $P^{-1} f=N A$.

We then write

$$
\begin{aligned}
I & =\text { ind } \lim P=\text { ind } \lim \left(P f^{-1}\right) f \\
& =\text { ind } \lim \left(\left(P^{-1} f\right)^{-1}\right) f \\
& =\text { ind } \lim \left((N A)^{-1}\right) f=\text { ind } \lim N^{-1} A f .
\end{aligned}
$$

As $N^{-1}$ is a torsion-free submodule of $K$ and $\mathrm{gl} \operatorname{dim} D=1, \mathrm{pd}_{D}\left(N^{-1}\right)<$ 1. Hence $\operatorname{pd}_{A} I<\operatorname{pd}_{D}\left(N^{-1}\right)<1$.

We may now state the main result.

(1.10) THEOREM. Let $M$ be a rank-one flat module over the Dedekind domain $D$, then

$$
\mathrm{gl} \operatorname{dim} S(M)=2 \text {. }
$$

Proof. By the previous discussion $M$ can be taken to be locally principal and, in determining the projective dimension of an ideal $I$ of $S(M)$, we may assume $I$ is neither flat nor finitely generated.

Let $J$ be the subideal of $I$ generated by "polynomials" of least degree. We have the exact sequence

$$
0 \rightarrow J \rightarrow I \rightarrow I / J \rightarrow 0
$$

and we show pd $J=\operatorname{pd} I / J=1$ giving pd $I=1$ and thus $\operatorname{gl} \operatorname{dim} A=2$.

If $J \cap D \neq 0$ then $I \cap D \neq 0$ and (1.6) gives $A /(I \cap D)$ is Noetherian and hence $I$ is finitely generated. Thus we may assume $J \cap D=0$. We claim $J$ is flat, and it is enough to check this at the primes of $D$.

If $p$ is a prime of $D, A_{p}=D_{p}[x]$ and

$$
J_{p}=g\left\{f_{1}, \ldots, f_{n}\right\}
$$

where $g$ is the gcd of the generators. If $g=1$ then $\left\{f_{1}, \ldots, f_{n}\right\}$ is a grade two ideal of $A_{p}$ and hence $\left\{f_{1}, \ldots, f_{n}\right\}=J_{p} \cap D_{p} \neq 0$ since, otherwise, $\left\{f_{1}, \ldots, f_{n}\right\}$ survives as a grade two ideal in the localization at $D_{p}-\{0\}$. But we assumed $J \cap D=0$ and so $g \neq 1$. In this case $\left\{f_{1}, \ldots, f_{n}\right\}$ has grade two and thus $\left\{f_{1}, \ldots, f_{n}\right\} \cap D_{p}=(\alpha) \neq 0$. This forces $\alpha g \in J_{p}$. As all the generators of $J_{p}$ have the same degree, this forces $g$ to be one of the generators. Thus $J_{p}=(g)$ and hence is flat. Thus $J$ is a flat ideal of $A=S(M)$ and $\mathrm{pd} J<1$ by (1.9). 
Next consider $I / J$. Since $(I / J) \otimes K=0, I / J$ is a torsion $D$-module. Thus

$$
I / J=\bigoplus_{p \in \text { spec } D} p \text {-primary components. }
$$

This decomposition is clearly valid as an $A$-module decomposition and the components are in fact $(I / J)_{p}$. Thus it is enough to find the projective dimension of $(I / J)_{p}$ and we first consider this as a $D_{p}[x]$-module and then make a change of rings. There is the $D_{p}[x]$-exact sequence

$$
0 \rightarrow J_{p} \rightarrow I_{p} \rightarrow(I / J)_{p} \rightarrow 0 \text {. }
$$

Since $J_{p}$ is finitely generated and flat, it is projective. As $g l \operatorname{dim} D_{p}[x]=2$, pd $I_{p}<1$ and we get $\mathrm{pd}_{D_{p}[x]}(I / J)_{p}<1$.

Since $(I / J)_{p}$ is $D_{p}$-torsion, some $d \in p$ kills $(I / J)_{p}$, i.e. $(d)_{p}=p^{n}$ and $d(I / J)_{p}=0$.

Let $p=p_{1}, \ldots, p_{n}$ be the primes of $D$ containing $d$ and consider the following change of rings

$$
D \rightarrow D_{\{d\}} \times D_{p_{1}} \times \cdots \times D_{p_{n}}
$$

where $D_{(d)}$ is the localization at the multiplicative powers of $d$. This extension is clearly faithfully flat and gives rise to

$$
A \rightarrow A_{\{d\}} \times A_{p_{1}} \times \cdots \times A_{p_{n}}=A^{\prime}
$$

which is also faithfully flat.

Now $(I / J)_{p} \otimes_{A} A^{\prime} \simeq(I / J)_{p}$ since $(I / J)_{p}=0$ in $A_{\{d\}}, A_{p_{2}} \cdots A_{p_{n}}$. Hence we make another application of faithfully flat descent to yield

$$
\operatorname{pd}_{A}(I / J)_{P}<1 \text {. }
$$

This being the case for each summand of $I / J$ we have $\mathrm{pd}_{A} I / J<1$ and the proof is complete.

2. The symmetric algebras of dimension two. In this section we consider the converse to Theorem (1.10). Specifically if $S_{A}(M)$ is symmetric algebra of global dimension two, what conditions are forced on the base ring $A$ and the module $M$ ? We begin with

(2.1) Lema. Every localization of $A$ at a prime ideal of $A$ is a domain.

Proor. Write

$$
B=S(M)=A+M+S^{2}(M)+\ldots
$$

and assume $A$ is a local ring. Let $a \in A$ and consider $a$ as an element of $B$, $a=(a, 0,0, \ldots)$. Now let $I=\left((0: a)_{A},(0: a)_{M}, \ldots\right)$ be the annihilator of $a$ in $B$. According to [11] $B$ is locally a domain, hence for any prime $P$ of $B$, $I_{p}=0$ or 1 . This forces $I$ to be a pure ideal of $B$. Hence $(0: a)_{A}$ is a pure ideal of $A$. But there are no nontrivial pure ideals in a local ring. Thus $(0: a)_{A}=0$ and $A$ is a domain. 
(2.2) Proposition. The algebra $B=S_{A}(M)$ is faithfully flat over the ring $A$.

Proor. We only need show that $M$ is a flat $A$-module and we check this at the maximal ideals of $A$. So assume $A$ is local and hence a domain. If $A$ is a field then $M$ is free. Otherwise the ideal

$$
I=M+S^{2}(M)+\ldots
$$

is a nonmaximal prime ideal. It follows easily from [11] that such ideals are flat. Thus $I \otimes B / I=I / I^{2}$ is $B / I$-flat. As $I / I^{2} \cong M$ and $B / I \cong A$ the result follows.

(2.3) COROLlary. The ring $A$ has global dimension at most two. If $S(M)$ is coherent, so is $A$.

Proof. By faithfully flat descent.

(2.4) Proposition. rk $M<2$ and $\mathrm{rk} M=2$ implies that some maximal ideal is also minimal.

Proof. First assume $A$ is local and not a field. If $K$ is the quotient field of $A$ then $\mathrm{gl} \operatorname{dim}\left(S_{A}(M) \otimes K\right)<2$. This forces $\mathrm{rk} M<2$.

If $\mathrm{rk} M=2$, then the ideal

$$
I=M+S^{2}(M)+\ldots
$$

is a nonmaximal prime and hence is flat. But $I_{A-\{0\}}=(x, y)$ in the ring $K[x, y]$ and $(x, y)$ is not a flat ideal, a contradiction.

This is as far as we may push the general analysis. Even in the case that $A$ is a domain we cannot eliminate the possibility that it has global dimension two. We will provide examples exhibiting the range of possibilities which reflect the lack of uniformity in the localization process. Rather than make any kind of uniformity assumptions, we shall just treat the case when $A$ is local of dimension two.

(2.5) THEOREM. If $A$ is a local ring of dimension two and $S(M)$ has dimension two, then $M=K$ the quotient field of $A$.

Proof. We have that $A$ is a domain and $M$ is flat, hence $S(M)$ is also a domain [8]. As $A$ is not a field rk $M=1$ and we may write

$$
B=S(M)=A+M t+M^{2} t^{2}+\ldots
$$

in a familiar fashion. We note that if $p$ is a nonmaximal prime ideal of $A$, the ideal $(p,(M t))$ is a nonmaximal prime of $B$ and hence is flat. Thus we have the flat ideal $(p,(M t))$ properly containing the prime ideal $(M t)$ and we apply (1.4) to get that $p M=M$ for all nonmaximal primes of $A$ and thus also for the maximal ideal $m$ of $A$. We now make a case by case analysis, assuming $M$ 
is infinitely generated. According to [11] there are three cases, and we adopt the terminology there.

Case I. $A$ is Noetherian, i.e. a regular local ring of dimension two. $A$ is then a U.F.D. and any $d \in A$ is uniquely a product of principal primes. Thus $d M=p_{1} \ldots p_{n} M=M$ and $M$ is a divisible rank one module and hence $M=K$.

Case II. $A$ is a valuation ring.

We shall show that if $M \neq K$ then $B=S(M)$ is not coherent and hence cannot have dimension two [10]. If $M \neq K$ there is an $a \in A$ such that $a M \neq M$. Pick $b \in M \backslash a M$. As $A$ is a valuation ring, the submodules of $M$ are linearly ordered. Thus $a M \subseteq(b)$ the submodule of $M$ generated by $b$. Consider then the ideals of $B$,

$$
B a=(a)+a M t+a M^{2} t^{2}+\ldots
$$

and

$$
B b t=b t+M b t^{2}+M^{2} b t^{3}+\ldots
$$

It is easy to see that $(B b t: B a)=(M t)$ which is not finitely generated. Hence $M=K$.

Case III. $A$ is an umbrella ring. In this case we have that $p_{\infty} A_{p_{\infty}}=p_{\infty}$ where $p_{\infty}$ is the distinguished ideal of $A$. Thus we have

$$
p_{\infty} M=M \text { and } p_{\infty} A_{p_{\infty}} M=p_{\infty} M_{\infty}=M_{\infty} .
$$

Hence $M=M_{\infty}$ the localization of $M$ at $p_{\infty}$. We then regard $M$ as an $A_{p_{\infty}}$-module. But $A_{p_{\infty}}$ is a valuation ring so we invoke Case II to find that $M=q f A_{p_{\infty}}=q f A=K$.

(2.6) REMARK. Greenberg [4] has shown that, under the proper cardinality conditions, the converse of (2.5) is true. That is, if $A$ is a local ring of dimension two, then $S_{A}(K)$ has global dimension two if $A$ has countably many principal prime ideals. This condition may fail when the ring $A$ is Noetherian, for example $A=C[x, y]_{(x, y)}$ where $C$ is the complex numbers. But if $A$ is either a valuation ring or an umbrella ring then there are only countably many.

(2.7) EXAMPLE. (2.5) provides us with a method of producing a ring which has Tor-dimension two but is not coherent.

Let $D$ be a valuation ring of dimension two with nonfinitely generated maximal ideal $m$. By [9] $m$ is countably generated and hence $S(m)$ is $n_{0}$-Noetherian. $S(m)$ has Tor-dimension two (1.3), but is not coherent. Thus its global dimension is three by [6].

As promised in the remark after (1.7), this example also shows that the Priffer assumption is not strong enough to guarantee coherence of the symmetric algebra. 
The reader is referred to [3] for similar examples, using the $D+M$ construction.

(2.8) EXAMPLE. We offer here an example of a symmetric algebra of dimension two which illustrates the difficulty of further classification.

Let $A=Q[x, y]_{(x, y) \cap(x-1)}$ where $Q$ is the rational numbers and the localization is with respect to the multiplicative set $Q[x, y] \backslash\{(x, y) \cup(x-1)\}$. Thus $A$ has exactly two maximal ideals. Setting $M=$ $\{f(x, y) / g(x, y) \mid g(x, y)$ is not divisible by $x-1\}$, we see that $M_{(x, y)}=$ $Q(x, y)$ and $M_{(x-1)}=(\alpha)$. As $A \rightarrow A_{(x, y)} \times A_{(x-1)}$ is faithfully flat the corresponding map $S(M) \rightarrow S(M)_{(x y)} \times S(M)_{(x-1)}$ is also faithfully flat, and we determine the dimension of $S(M)$ in the two components.

$S(M)_{(x, y)}$ has only countably many principal primes and thus its dimension is two as noted in Remark (2.6). As for $S(M)_{(x-1)}$ we note that $A_{(x-1)}$ is a D.V.R. and we invoke the syzygies theorem.

Thus we take the point of view that the algebras of section one are the mainstream examples of symmetric algebras of dimension two and that the others should be regarded as exceptions.

\section{BIBLIOGRAPHY}

1. N. Bourbaki, Algebra. I, Addison-Wesley, Reading, Mass., 1974.

2. __ Algébre commutative, Chapters I, II, Hermann, Paris, 1961.

3. D. Dobbs, On the global dimension of $D+M$ (preprint).

4. B. Greenberg, Global dimension of cartesian squares, J. Algebra 32 (1974), 31-43. $1-89$.

5. L. Gruson and M. Raynaud, Critères de platitude et de projectioite, Invent. Math. 13 (1971),

6. C. U. Jensen, Homological dimension of rings with countably generated ideals, Math. Scand. 18 (1966), 97-105.

7. I. Kaplansky, Commutative rings, Allyn and Bacon, Boston, Mass., 1970.

8. D. Lazard, Autour de la platitude, Bull. Soc. Math. France 97 (1969), 81-128.

9. B. Osofsky, Global dimension of valuation rings, Trans. Amer. Math. Soc. 126 (1967), 136-149.

10. H. Sheng, Finiteness in prime ideals in rings of global dimension two, Proc. Amer. Math. Soc. 41 (1973), 363-369.

11. W. Vasconcelos, The local rings of global dimension two, Proc. Amer. Math. Soc. 35 (1972), 381-386.

12. $\longrightarrow$ Rings of global dimension two, Proc. Conf. Comm. Algebra, Lecture Notes in Math., vol. 311, Springer-Verlag, Berlin and New York, 1973.

Departament of Mathematics, Rutoers University, New Brunswick, NeW Jersey 08903

Current address: Department of Mathematics, George Mason University, Fairfax, Virginia 22030 